\title{
Cucurbitacin B enhances apoptosis in gefitinib resistant non-small cell lung cancer by modulating the miR-17-5p/STAT3 axis
}

\author{
BAODAN YU ${ }^{1}$, LIXIA ZHENG ${ }^{2}$, HUIQIN TANG ${ }^{1}$, WEIXIN WANG ${ }^{1}$ and YONGPING LIN ${ }^{1}$ \\ Departments of ${ }^{1}$ Laboratory Medicine and ${ }^{2}$ Hematology, The First Affiliated Hospital of \\ Guangzhou Medical University, Guangzhou, Guangdong 510120, P.R. China
}

Received January 25, 2021; Accepted July 20, 2021

DOI: $10.3892 / \mathrm{mmr} .2021 .12349$

\begin{abstract}
Tyrosine kinase inhibitors, such as gefitinib, are currently widely used as targeted therapeutics for non-small cell lung cancer (NSCLC). Although drug resistance has become a major obstacle to successful treatment, mechanisms underlying resistance to gefitinib remain unclear. Therefore, the present study aimed to investigate the impact of adjunctive cucurbitacin $\mathrm{B}(\mathrm{CuB})$ on gefitinib resistance $(\mathrm{GR})$ in the PC9 cell line, including identifying underlying mechanisms. Reverse transcription-quantitative PCR demonstrated significant downregulation of microRNA (miR)-17-5p expression in GR PC9 cells (PC9/GR), and this could be reversed by $\mathrm{CuB}$. During combination treatment with $\mathrm{CuB}$ and gefitinib at $\mathrm{IC}_{25}$, PC9/GR cell proliferation was downregulated, and apoptosis was upregulated. The presence of a miR-17-5p inhibitor negated the effects of $\mathrm{CuB}$ and gefitinib, whereas the presence of a miR-17-5p mimic enhanced them. Luciferase assays demonstrated that the hypothetical target gene, signal transducer and activator of transcription 3 (STAT3), was directly targeted by miR-17-5p. Moreover, significant elevation of the STAT3 protein and phosphorylation levels in PC9/GR cells was reversed by the addition of $\mathrm{CuB}$, despite a lack of change in STAT3 transcription level. During combined treatment with $\mathrm{CuB}$ and gefitinib at $\mathrm{IC}_{25}$, the STAT3 protein expression was negatively associated with the expression of miR-17-5p.
\end{abstract}

Correspondence to: Dr Yongping Lin, Department of Laboratory Medicine, The First Affiliated Hospital of Guangzhou Medical University, 151 Yanjiang West Road, Yuexiu, Guangzhou, Guangdong 510120, P.R. China

E-mail: 18928868278@163.com

Abbreviations: $\mathrm{CuB}$, cucurbitacin B; RT-qPCR, reverse transcription-quantitative polymerase chain reaction; NSCLC, non-small cell lung cancer; IC, inhibitory concentration; NC, negative control; UTR, untranslated region; STAT3, signal transducer and activator of transcription 3; SDS, sodium dodecyl sulfate; PVDF, polyvinylidene fluoride; BSA, bovine serum albumin

Key words: microRNA-17-5p, signal transducer and activator of transcription 3, cucurbitacin B, gefitinib, non-small cell lung cancer, treatment resistance
Overexpression of STAT3 increased proliferation and decreased apoptosis and the protein levels of apoptosis-related factors cleaved caspase-3 and cleaved caspase-9 of PC9/GR cells. Findings indicated that STAT3 protein and phosphorylation levels became elevated in response to gefitinib, and that CuB-induced miR-17-5p expression led to STAT3 degradation, thereby ameliorating GR. In summary, $\mathrm{CuB}$ reduced the proliferation of GR PC9 cells by modulating the miR-17-5p/STAT3 axis, and may represent a promising potential novel strategy for the reversal of GR.

\section{Introduction}

Lung cancer is the leading cause of cancer-related mortality worldwide, and non-small cell lung cancer (NSCLC) is the most common type of lung cancer (1). Tyrosine kinase inhibitors, such as gefitinib, are widely used as targeted therapeutics for the treatment of NSCLC (2-4). Although most patients with NSCLC initially respond to chemotherapy, their cancer gradually develops resistance, leading to cancer progression, or recurrence and poorer prognoses (5). Therefore, there is an urgent need to identify an appropriate agent that could be combined with gefitinib to effectively combat gefitinib resistance (GR). However, most agents that have been combined with gefitinib, such as pemetrexed (6), thalidomide (7) or metformin (8), have variable degrees of toxicity and several side effects.

Cucurbitacin $\mathrm{B}(\mathrm{CuB})$ is an oxidized tetracyclic triterpenoid derived from plants of the Cucurbitaceae family. This molecule exhibits antineoplastic activity in various types of cancer $(9,10)$, with low toxicity and fewer side effects (9). In addition, $\mathrm{CuB}$ can activate the protein expression of apoptosis-related factors cleaved caspase-3 and cleaved caspase-9 (11), inhibit the metastatic ability of NSCLC (12) and inhibit 4-(methylnitros amino)-1-(3-pyridyl)-1-butanone-induced lung tumors (13). In addition, Liu et al (5) found that $\mathrm{CuB}$ inhibited GR NSCLC by inducing lysosomal degradation of epidermal growth factor receptor. Therefore, gefitinib is a candidate for combination therapy.

It is necessary to explore the mechanisms of action of combined drug therapy for NSCLC. MicroRNAs (miRNAs/miRs) are a type of small non-coding RNA. Dysregulation of specific miRNAs may be involved in the development of resistance to a variety of cancer treatments (i.e., modulating cancer cell sensitivity 
to such therapies) $(14,15)$. One such miRNA, miR-17-5p, is closely associated with the occurrence, progression and prognosis of lung cancer (16-19). miRNAs can modulate the expression of target genes at both the transcriptional and post-transcriptional levels (20). In the present study, the TargetScan database was analyzed and it was found that among the putative target genes, the signal transducer and activator of transcription 3 (STAT3) gene is a potential target for miR-17-5p.

STAT3 is a transcription factor known to promote tumorigenesis (21). It is frequently activated in pre-neoplastic and cancerous cells and is frequently identified during research studies as being dysregulated in lung cancer (22-24). Activation of STAT3 can promote proliferation and metastasis of NSCLC cells (25). Studies have shown that miRNAs, such as miR-34a and miR-519a, can inhibit the process of NSCLC by regulating STAT3 expression $(26,27)$. In addition, some studies have shown that miR-17-5p can target STAT3 to reduce the activity of rat cardiomyocytes (28), or promote the apoptosis of breast cancer cells (29), and this antagonistic regulatory relationship between miR-17-5p and STAT3 has been confirmed. However, the mechanism by which the miR-17-5p/STAT3 axis regulates $\mathrm{CuB}$ to inhibit NSCLC progression remains unclear.

In the present study, GR PC9 cells were cultured in vitro to simulate GR in patients with lung cancer, and the role of the miR-17-5p/STAT3 axis in regulating the effect of $\mathrm{CuB}$ in GR NSCLC cells was explored from the perspective of CuB-miRNA-mRNA interaction.

\section{Materials and methods}

Reagents, cells and biomolecules. CuB (cat. no. C8499) and gefitinib (cat.no. SML1657) were purchased from Sigma-Aldrich (Merck KGaA). A human lung adenocarcinoma-derived cell line(PC9) was purchased from ProcellLife Science \& Technology Co., Ltd., and 293T cell lines were purchased from the American Type Culture Collection. Roswell Park Memorial Institute (RPMI)-1640 medium, phosphate-buffered saline (PBS) and fetal bovine serum (FBS) were purchased from Gibco (Thermo Fisher Scientific, Inc.). A PrimeScript RT reagent kit and SYBR Premix ExTaq II kit were purchased from Takara Biotechnology Co., Ltd. Lysis buffer and ECL reagent were purchased from Thermo Fisher Scientific, Inc. A BCA protein assay kit was purchased from Tiangen Biotech Co., Ltd. Polyvinylidene fluoride (PVDF) membranes were purchased from MilliporeSigma. Sodium dodecyl sulfate (SDS), bovine serum albumin (BSA) blocking buffer $(5 \%)$ and trypsin-EDTA $(0.25 \%)$ solution and trypan blue staining solution $(0.4 \%)$ were purchased from Beijing Solarbio Science \& Technology Co., Ltd. Anti-STAT3 (cat. no. ab68153), anti-phosphorylated (p)-STAT3 (cat. no. ab267373), anti-caspase-3 (cat. no. ab32351), anti-cleaved caspase-3 (cat. no. ab2302), anti-caspase-9 (cat. no. ab32539), anti-cleaved caspase-9 (cat. no. ab2324), anti-GAPDH antibodies (cat. no. ab181602) and goat anti-rabbit (cat. no. ab205718) were purchased from Abcam. Guangzhou RiboBio Co., Ltd., synthesizedamiR-17-5pmimic(5'-CAAAGUGCUUACAGUGC AGGUAG-3'), miR-17-5p scrambled mimic negative control (NC; 5'-ACUAAUGAGCGAGUGAAUCCGUG-3'), miR-17-5p inhibitor (5'-CTACCTGCACTGTAAGCACTTTG-3') and miR-17-5p scrambled inhibitor NC (5'-CAGUACUUUUGUG UAGUACAA-3'). Sangon Biotech Co., Ltd., synthesized a
STAT3-encoding plasmid (ov-STAT3), a corresponding NC plasmid (ov-NC) and PCR primer pairs targeting STAT3, GAPDH, miR-17-5p and U6. TRIzol ${ }^{\circledR}$, Lipofectamine ${ }^{\circledR} 2000$ transfection reagent and the SYBR-Green I Real-Time PCR kit were purchased from Invitrogen (Thermo Fisher Scientific, Inc.). A Cell Counting Kit-8 (CCK-8) and RIPA lysis buffer were purchased from Beyotime Institute of Biotechnology. An Annexin V-FITC Apoptosis Detection Kit was purchased from BD Biosciences. The wild-type (WT) and mutant type (MUT) 3'-UTR of STAT3 were synthesized (Guangzhou RiboBio Co., Ltd.) and inserted into psiCHECK ${ }^{\mathrm{TM}}-2$ vector plasmids and pRL-SV40 reporter vector plasmids (all plasmids were purchased from Promega Corporation). psiCHECK-2 plasmids carried two reporter genes (Renilla and firefly luciferases), whereas pRL-SV40 plasmids carried only one (Renilla luciferase).

Culture conditions for PC9 or PC9/GR cells, induction of GR and transfection with miRNAs. The PC9 or PC9/GR cell lines were cultured at $37^{\circ} \mathrm{C}$ and $5 \% \mathrm{CO}_{2}$ in RPMI-1640 medium supplemented with $10 \%$ FBS (hereafter referred to as normal culture medium). To produce a GR cell variant (PC9/GR), gefitinib concentration was progressively increased, as previously described (30). Thereafter, resistance was maintained by including $1 \mu \mathrm{g} / \mathrm{ml}$ gefitinib in culture media. Once cells reached 60-80\% confluence, they were transfected with $50 \mathrm{nM}$ ov-STAT3, $100 \mathrm{nM}$ miR-17-5p mimic, mimic NC, inhibitor or inhibitor $\mathrm{NC}$ using Lipofectamine 2000 liposomes at $37^{\circ} \mathrm{C}$, according to the manufacturer's instructions. Cells were incubated for $4 \mathrm{~h}$, medium was replaced with normal cell culture medium, and cells were incubated for a further $48 \mathrm{~h}$ prior to analysis.

Confirmation of induced PC9/GR drug resistance and evaluation of the impact of $\mathrm{CuB}$. Following incubation of PC9 and PC9/GR cells with varying concentrations of gefitinib $(0,0.39$, $0.78,1.56,3.125,6.25,12.5,25$ and $50 \mu \mathrm{M})$ and $\mathrm{CuB}(2,4,6$, $8,10,12$ and $16 \mu \mathrm{g} / \mathrm{ml}$ ) for $48 \mathrm{~h}$, the number of viable cells was determined via CCK-8 (according to the manufacturer's instructions). Concentrations of gefitinib that produced $50 \%$ inhibitory concentration $\left(\mathrm{IC}_{50}\right)$ were used to calculate the drug resistance index $(\mathrm{RI}): \mathrm{RI}=\left(\mathrm{IC}_{50}\right.$ for $\mathrm{P} 9 / \mathrm{GR}$ cells $) /\left(\mathrm{IC}_{50}\right.$ for $\mathrm{PC} 9$ cells). PC9/GR cells were cultured at $\mathrm{IC}_{25}$ of $\mathrm{CuB}$ or gefitinib.

Reverse transcription-quantitative PCR (RT-qPCR) assay. Total RNA was extracted from PC9 cells, PC9/GR cells incubated in the presence or absence of varying concentrations of $\mathrm{CuB}(2,4,6,8,10,12$ and $16 \mu \mathrm{g} / \mathrm{ml})$ and PC9/GR cells treated with a combination of $\mathrm{CuB}$ and gefitinib at $\mathrm{IC}_{25}$ using TRIzol reagent, according to the manufacturer's instructions. Extracted RNA was reverse transcribed to cDNA using a PrimeScript RT reagent kit, according to the manufacturer's instructions. The RT-qPCR procedure was performed using a SYBR Premix ExTaq II kit (according to the manufacturer's instructions) in conjunction with a 7500 Real-Time PCR System (Applied Biosystems; Thermo Fisher Scientific, Inc.). Thermocycling conditions were as follows: $95^{\circ} \mathrm{C}$ for $5 \mathrm{~min}$, followed by 40 cycles of $95^{\circ} \mathrm{C}$ for $15 \mathrm{sec}$ and $60^{\circ} \mathrm{C}$ for $1 \mathrm{~min}$. Primer pair sequences were as follows: STAT3 forward, 5'-ATCCTGAAG CTGACCCAGG-3' and reverse, 5'-CTGCAGGTCGTTGGTG TCA-3'; GAPDH forward, 5'-GCTCATTTGCAGGGGG GAG-3' and reverse, 5'-GTTGGTGGTGCAGGAGGCA-3'; 
miR-17-5p forward, 5'-ACACTCCAGCTGGGCAAAGTGC TTACAGTGC-3' and reverse, 5'-CTCAACTGGTGTCGT GGA-3'; and U6 forward, 5'-CTCGCTTCGGCAGCACA-3' and reverse, 5'-AACGCTTCACGAATTTGCGT-3'. Target RNA levels were normalized to those of housekeeping genes $G A P D H$ or $U 6$. Relative mRNA expression levels were calculated using the $2^{-\Delta \Delta C q}$ method (31).

Western blotting. PC9 or PC9/GR cells were lysed using ice-cold RIPA lysis buffer. Lysate protein concentrations were determined using a BCA protein assay kit. Equal amounts of denatured proteins $(20 \mu \mathrm{g})$ were resolved via $10 \%$ SDS-polyacrylamide gel electrophoresis and separated proteins were subsequently transferred to a PVDF membrane. The membrane was blocked with 5\% BSA for $1 \mathrm{~h}$ at $20^{\circ} \mathrm{C}$, incubated with primary antibodies (anti-STAT3, 1:1,000; anti-p-STAT3, 1:1,000; anti-caspase-3, 1:5,000; anti-caspase-3, 1:5000; anti-cleaved caspase-3, 1:500; anti- caspase-9, 1:2,000 and anti-cleaved caspase- $9,1 \mu \mathrm{g} / \mathrm{ml}$ ) overnight at $4^{\circ} \mathrm{C}$, rinsed with TBS containing $0.05 \%$ Tween-20 buffer (Beijing Solarbio Science \& Technology Co., Ltd.) twice for $10 \mathrm{~min}$ each time, and subsequently incubated with the horseradish peroxidase-conjugated secondary antibody (goat anti-rabbit; $1: 10,000$ ) for $2 \mathrm{~h}$ at $23 \pm 2^{\circ} \mathrm{C}$. Protein bands were visualized by the addition of ECL reagent in conjunction with an imaging system (DNR Bio-Imaging Systems, Ltd.). Anti-GAPDH antibody $(1: 10,000)$ was used as a loading control. Image J software (version 1.49n; National Institutes of Health) was used for densitometry.

Proliferation assay. A single-cell suspension was prepared via trypsinization of PC9 or PC9/GR cells, and these cells were seeded into six-well plates (Costar; Corning, Inc.) at a density of 500 cells per well in $2 \mathrm{ml}$ culture medium. After cells were cultured for 2 weeks, stained with trypan blue staining solution $\left(23 \pm 2^{\circ} \mathrm{C}\right.$ for $\left.5 \mathrm{~min}\right)$ and counted. Survival rates were determined via CCK-8, using 96-well plates (cat. no. 3599; Costar; Corning, Inc.), according to the manufacturer's instructions. Briefly, cells were seeded at a density of $5 \times 10^{3}$ cells/well and cultured for 24-48 h, $10 \mu \mathrm{l}$ CCK-8 solution was added per well, plates were incubated for $60 \mathrm{~min}$ at $23 \pm 2^{\circ} \mathrm{C}$ and absorbance at $450 \mathrm{~nm}$ was measured using an enzyme-labeled instrument (Multiskan MK3, Thermo Fisher Scientific, Inc.). The $\mathrm{IC}_{50}$ and $\mathrm{IC}_{25}$ values are obtained by analyzing the survival rate of $\mathrm{PC} 9$ or PC9/GR cells by GraphPad Prism 8 (GraphPad Software Inc.).

Apoptosis assay. Early + late apoptosis of PC9 or PC9/GR cells was assessed using an Annexin V-FITC Apoptosis Detection Kit, according to the manufacturer's instructions. Briefly, cells $\left(1 \times 10^{6}\right.$ cells $\left./ \mathrm{ml}\right)$ were harvested by trypsin digestion, washed twice using ice-cold PBS and resuspended in $500 \mu$ l binding buffer. Next, cells were incubated with $5 \mu$ l Annexin V-FITC and $5 \mu \mathrm{l}$ propidium iodide (PI) in the dark for $15 \mathrm{~min}$ at $23 \pm 2^{\circ} \mathrm{C}$, followed by flow cytometry (BD FACSCalibur; BD Biosciences); FlowJo software (version 10.6.2; FlowJo LLC) was used for analysis.

Binding site prediction. The TargetScan database 7.2 (http://www.targetscan.org/vert_72/) was used to predict STAT3 binding sites for miR-17-5p.
Dual-luciferase reporter assay. 293T cells were cultured in Dulbecco's modified Eagle's medium (Gibco; Thermo Fisher Scientific, Inc.) supplemented with 10\% FBS. Thereafter, 293T cells were transfected with $500 \mathrm{ng}$ each of a miR-17-5p mimic or inhibitor and their NCs, $1 \mu \mathrm{g}$ each of the vector plasmid containing WT or MUT STAT3, and $50 \mathrm{ng}$ pRL-SV40 reporter vector plasmid using Lipofectamine ${ }^{\circledR} 2000$ (Invitrogen; Thermo Fisher Scientific, Inc.). Cells were incubated for $48 \mathrm{~h}$, and luciferase activity was measured using a Dual-Luciferase Reporter Assay System (Promega Corporation) according to the manufacturer's instructions. Briefly, absorbance at $490 \mathrm{~nm}$ (determined by luciferase activity) was measured, and target values were calculated with respect to the NC groups. The ratio of firefly to Renilla activity was used to normalize firefly luciferase values.

Statistical analysis. All experiments were performed in triplicate. All data are expressed as the mean \pm standard deviation. All statistical analyses were performed using SPSS version 21.0 statistical analysis package (IBM Corp.). Comparison of multiple groups was performed using one-way ANOVA followed by Dunnett's post hoc test. Means of two groups were compared using an unpaired Student's t-test. $\mathrm{P}<0.05$ was considered to indicate a statistically significant difference.

\section{Results}

Gefitinib-induced downregulation of miRNA expression. The differences in the expression levels of various miRNAs in PC9 and PC9/GR cells were detected via RT-qPCR. The results showed that miR-17-92 family levels were downregulated in all PC9/GR cells compared with PC9 cells. Among them, miR-17-5p downregulation was the most significant, so in this study, miR-17-5p was selected for further study (Fig. 1A).

Confirmation of induced PC9/GR drug resistance. Survival rates of PC9 and PC9/GR cells following exposure to gefitinib were determined (Fig. 1B). Inhibitory concentrations of gefitinib were as follows: $\mathrm{IC}_{50}$ for PC9 cells, $3.89 \mu \mathrm{g} / \mathrm{ml}$; $\mathrm{IC}_{25}$ for PC9/GR cells, $10.4 \mu \mathrm{g} / \mathrm{ml}$; and $\mathrm{IC}_{50}$ for PC9/GR cells, $20.6 \mu \mathrm{g} / \mathrm{ml}$. A calculated RI of 5.3 indicated successful induction of moderate GR in PC9/GR cells.

Addition of $C u B$ ameliorates the gefitinib-induced downregulation of $m i R-17-5 p$ expression. RT-qPCR results showed that the expression of miR-17-5p in PC9/GR cells was gradually upregulated with a gradual increase of $\mathrm{CuB}$ concentration (Fig. 1C).

Effect of CuB on gefitinib-induced resistance in PC9/GR cells. The CCK- 8 assay showed that the $\mathrm{IC}_{25}$ of $\mathrm{CuB}$ on PC9/GR cells was $2.6 \mu \mathrm{g} / \mathrm{ml}$. (Fig. 1D). Then, the effect of the combined action of $\mathrm{CuB}\left(\mathrm{IC}_{25}\right)$ and gefitinib $\left(\mathrm{IC}_{50}\right)$ on PC9/GR cells was analyzed. The results showed that the combination of $\mathrm{CuB}$ and gefitinib significantly increased apoptosis (Fig. 1E and F) and reduced survival rate (Fig. 1G) in PC9/GR cells compared with $\mathrm{CuB}\left(\mathrm{IC}_{25}\right)$ or gefitinib $\left(\mathrm{IC}_{50}\right)$ treatment alone.

Effect of $C u B$ on miR-17-5p expression and cell proliferation/apoptosis at gefitinib $I C_{25}$ in PC9/GR cells transfected 
A

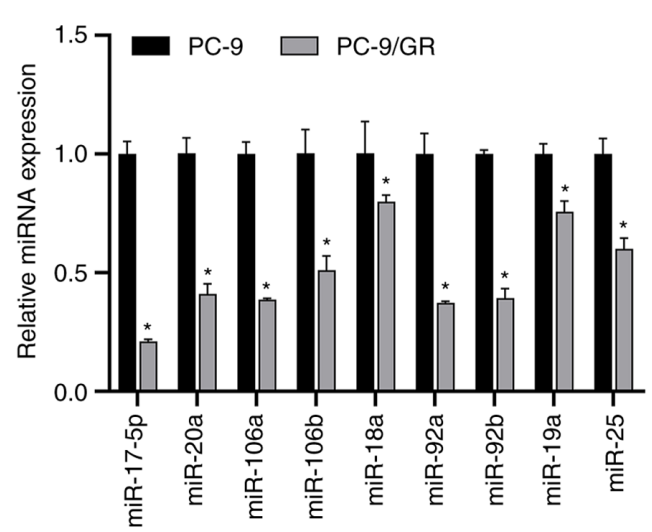

C

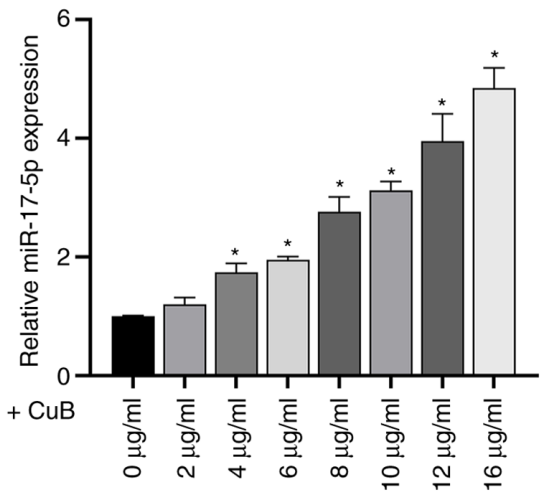

B

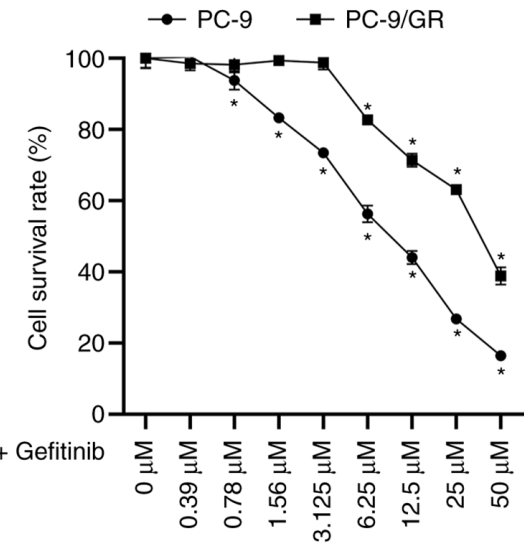

D

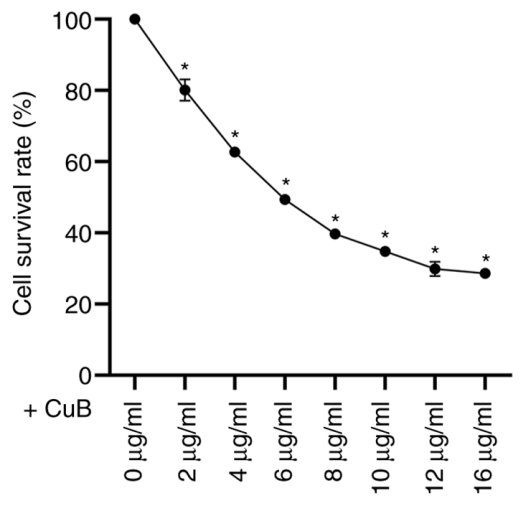

E

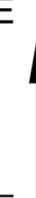

$\bar{\alpha}$

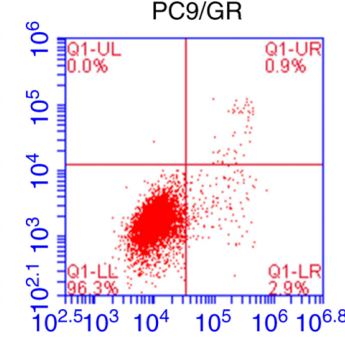

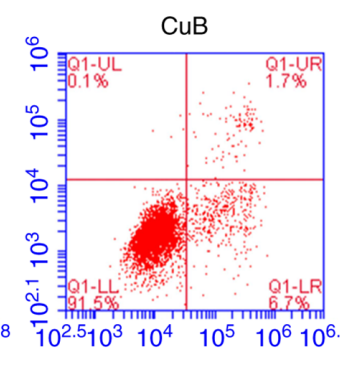
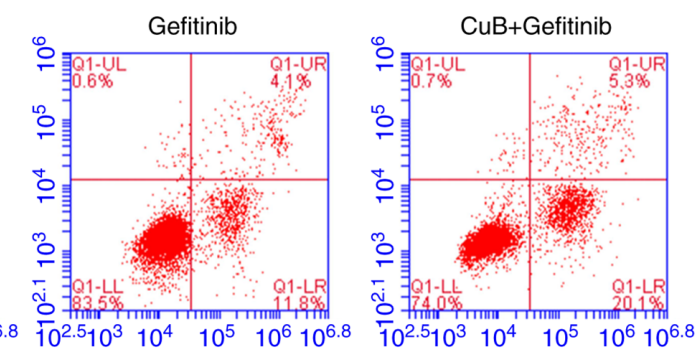

Annexin V FITC

$\mathrm{F}$

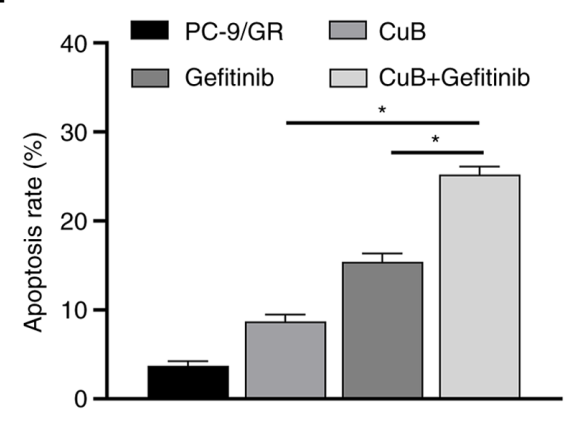

G

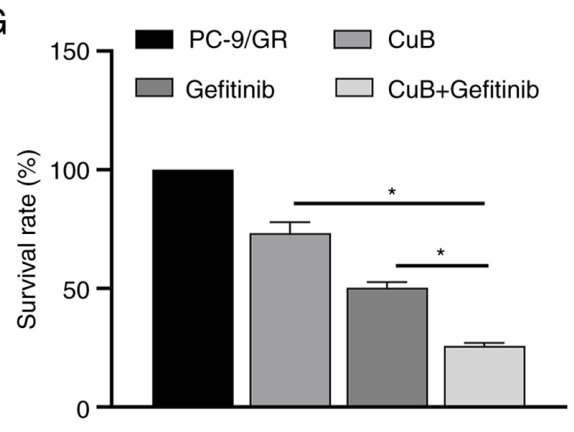

Figure 1. Effect of gefitinib, with or without $\mathrm{CuB}$, on PC9 cells. (A) Expression levels of miR-17-92 family in PC9 and PC9/GR cells. "P<0.05 vs. PC-9. (B) Survival rates of PC9 and PC9/GR cells. (C) Impact of differing CuB concentrations on expression levels of miR-17-5p in PC9/GR cells. (D) Impact of differing CuB concentrations on survival rate of PC9/GR cells. "P<0.05 vs. $0 \mu \mathrm{g} / \mathrm{ml}$. (E and F) Impact of CuB/gefitinib on apoptosis of PC9/GR cells. (G) Impact of CuB/gefitinib on survival rate of PC9/GR cells. All values are expressed as mean \pm standard deviation. "P<0.05. CuB, cucurbitacin B; miR, microRNA; GR, gefitinib-resistant.

with miR-17-5p mimic or inhibitor. RT-qPCR investigating the effect of CuB on miR-17-5p expression in PC9/GR cells transfected with miRNAs confirmed that miR-17-5p expression levels were lower in the miR-17-5p inhibitor group compared with in the inhibitor NC group, whereas levels were higher in the miR-17-5p mimic group compared with the NC mimic group (Fig. 2A). This result demonstrated that the synthetic miR-17-5p mimics and inhibitors were effective. Additionally, 
A

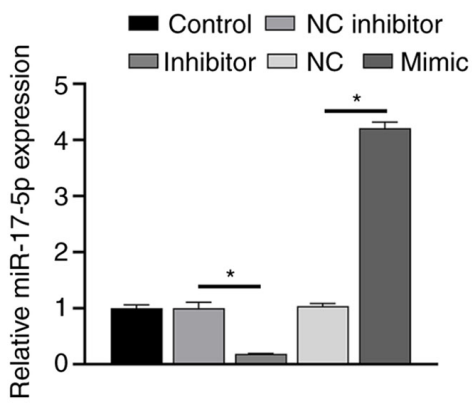

$\mathrm{B}$

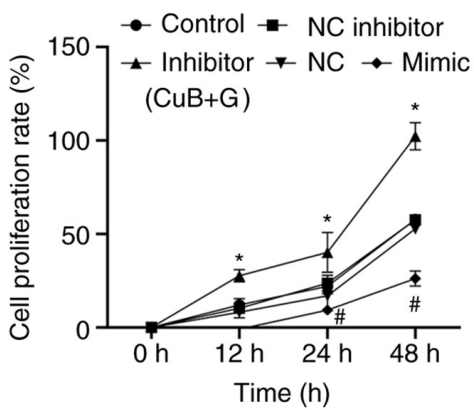

C

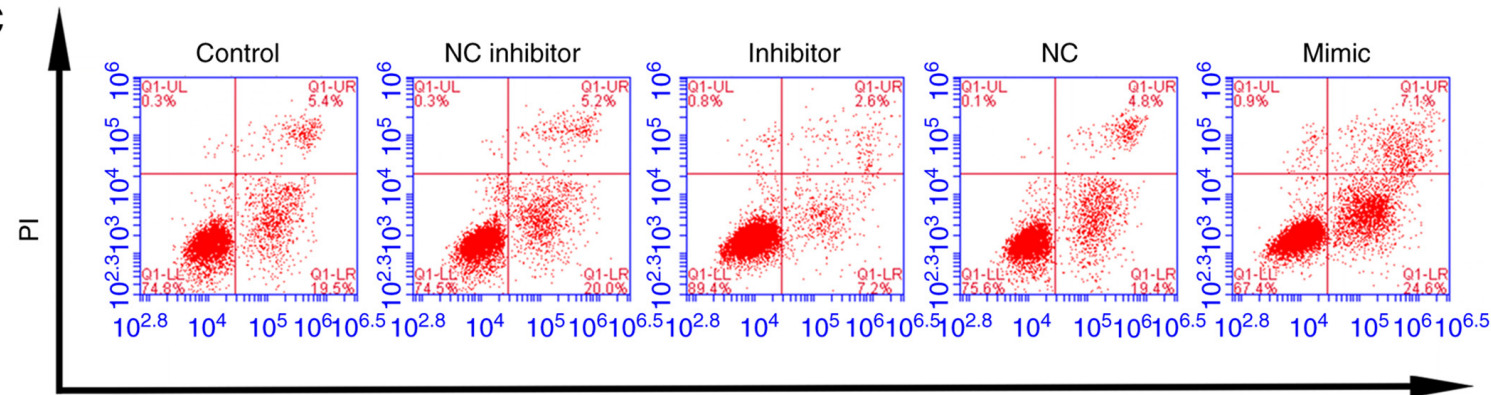

Annexin V FITC
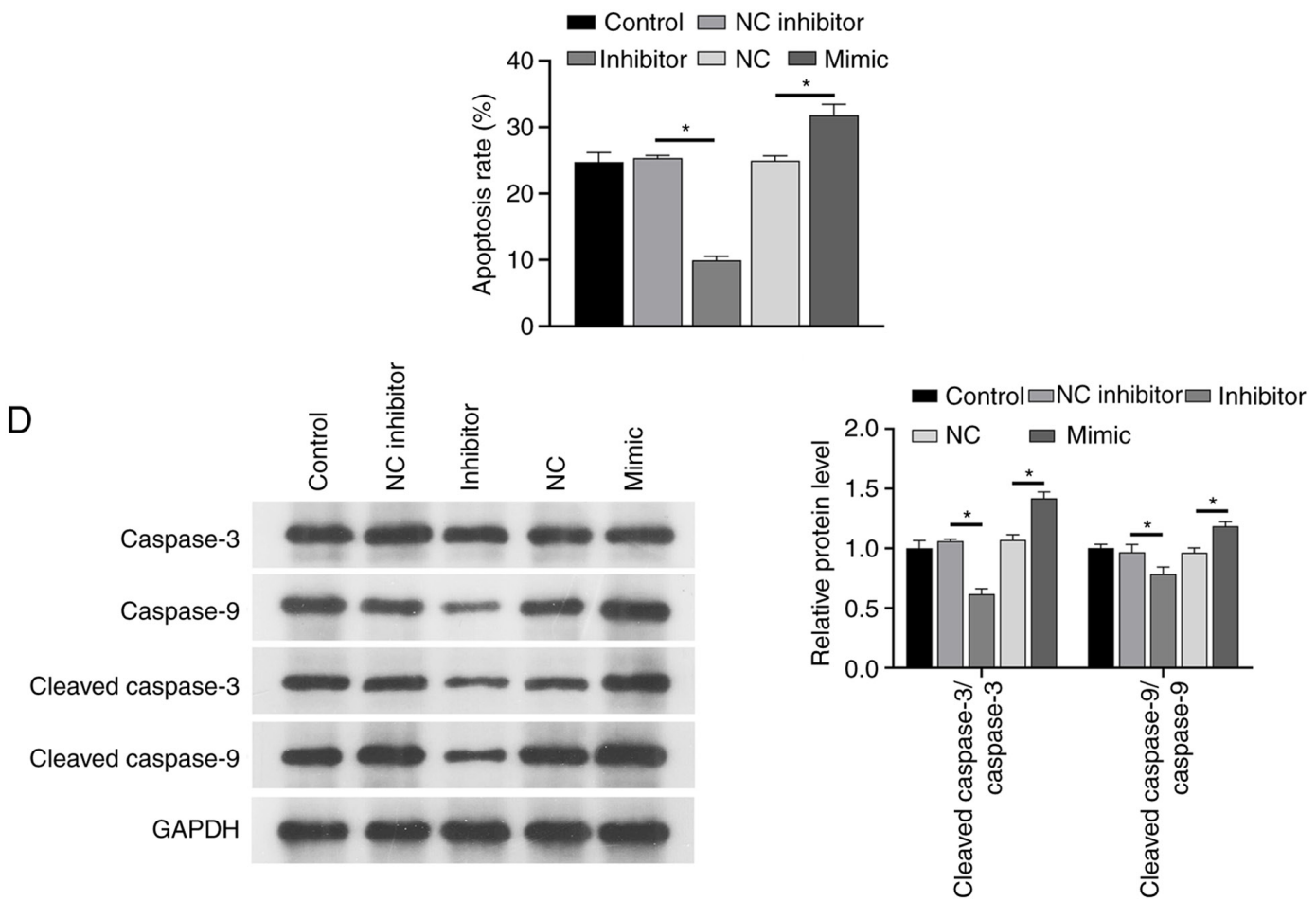

Figure 2. Effect of $\mathrm{CuB}$ in the presence of gefitinib at $\mathrm{IC}_{25}$ on PC9/GR cells transfected with miR-17-5p mimic or inhibitor. (A) Transcript levels of miR-17-5p. (B) Cell proliferation rates. (C) Cell apoptosis rates. (D) Protein expression levels of caspase-3, cleaved caspase-3, caspase-9 and cleaved caspase-9. All values are expressed as mean \pm standard deviation. ${ }^{*, *} \mathrm{P}<0.05$. CuB, cucurbitacin $\mathrm{B}$; miR, microRNA; NC, negative control; GR, gefitinib-resistant.

the results of CCK-8, flow cytometry and western blotting showed that the miR-17-5p inhibitor promoted the proliferation of PC-9/GR cells and inhibited apoptosis and protein levels of cleaved caspase- 3 and cleaved caspase- 9 under the combined action of $\mathrm{CuB}$ and gefitinib (Fig. 2B-D). Transfection with the miR-17-5p mimic induced the opposite effects of those noted for the miR-17-5p inhibitor.

STAT3 is directly targeted by miR-17-5p. Since miRNAs usually inhibit protein synthesis by binding to the 3 '-UTR region of mRNA, the putative downstream target genes of miR-17-5p were analyzed using the TargetScan database. The results showed that the oncogene STAT3, which has been demonstrated by a number of studies to play a key role in the development of NSCLC $(25,32)$, is also one of the assumed target genes of miR-17-5p, so STAT3 was selected in this study for further study into the molecular mechanism of this miRNA. The TargetScan database analysis predicted the putative miR-17-5p binding site of the STAT3 oncogene (Fig. 3A). The dual-luciferase reporter assay showed that luciferase activity was significantly downregulated after transfection of miR-17-5p mimics in STAT3 3'-UTR group compared with NC mimic group. Meanwhile, 


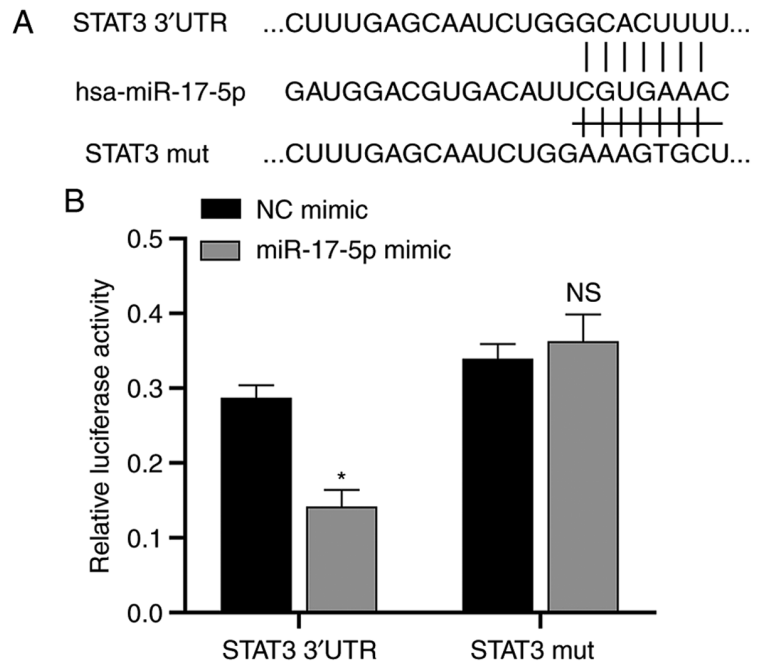

Figure 3. STAT3 gene is a direct target of miR-17-5p. (A) Prediction of putative STAT3 gene binding sites for miR-17-5p. (B) Direct binding of miR-17-5p to STAT3 gene. All values are expressed as mean \pm standard deviation. "P $<0.05$ vs. NC mimic. NS, not significant; miR, microRNA; mut, mutant.

there was no significant difference in luciferase activity after the transfection of mimics with the MUT STAT3 compared with the NC mimic group. This result demonstrated that STAT3 was directly targeted by miR-17-5p (Fig. 3B).

Effect of $C$ C B on STAT3 transcription and protein expression levels. The RT-qPCR assay demonstrated that GR did not significantly alter STAT3 transcription in PC9/GR cells (Fig. 4A), whereas western blotting demonstrated that it significantly upregulated STAT3 expression levels and promoted the phosphorylation of STAT3 (P<0.05; Fig. 4B). Then, RT-qPCR and western blotting confirmed that the STAT3 transcript level was not significantly altered by $\mathrm{CuB}$ ( $\mathrm{P}>0.05$; Fig. $4 \mathrm{C})$, but $\mathrm{CuB}$ decreased STAT3 protein expression and inhibited STAT3 phosphorylation in a dose-dependent manner (Fig. 4D).

miR-17-5p/STAT3 axis is modulated by $C u B$ and gefitinib combination treatment. In the presence of $\mathrm{IC}_{25}$ concentrations of $\mathrm{CuB}$ and gefitinib, RT-qPCR and western blotting assays investigating the impact of $\mathrm{CuB}$ on gefitinib sensitivity of PC9/GR cells transduced with miRNAs demonstrated that while the CuB-induced change in miR-17-5p expression did not significantly alter STAT3 transcript level (Fig. 5A), STAT3 protein and phosphorylation levels were lower in the miR-17-5p mimic group than in the mimic NC group, and higher in the miR-17-5p inhibitor group than in the inhibitor NC group (Fig. 5B). Furthermore, transfection with ov-STAT3 increased the expression levels of the STAT3 transcript (Fig. 5C), STAT3 and p-STAT3 protein expression levels (Fig. 5D), and negated the beneficial effects of $\mathrm{CuB}$ on gefitinib sensitivity in PC9/GR cells, including restoring increased proliferation (Fig. 5E) and decreased apoptosis (Fig. 5F) and protein levels of cleaved caspase-3 and cleaved caspase-9 (Fig. 5G) compared with the ov-NC group. These results confirmed that overexpression of STAT3 could reverse the apoptotic effect of $\mathrm{CuB}$ on PC9/GR cells and increase cell proliferation. Briefly, these results indicated that $\mathrm{CuB}$ regulated the miR-17-5p/STAT3 axis to increase gefitinib sensitivity in PC9/GR cells.

\section{Discussion}

Multiple miRNAs, including the miR-17-92 family have been implicated in various types of malignancies $(33,34)$. Abnormal expression of miR-17-92 family members is closely associated with the occurrence and progression of lung cancer $(35,36)$. The present study investigated whether one miR-17-92 family member, miR-17-5p, may be involved in modulating NSCLC drug sensitivity. The findings demonstrated that, compared with gefitinib-sensitive PC9 parent cells, PC9/GR cells expressed lower levels of miR-17-5p, suggesting that miR-17-5p may play a role in GR. This result is similar to the findings of a study conducted by Gong et al (37) on miR-17-5p regulating GR in A549 cells. As miRNAs typically regulate cellular functions through interaction with target genes (38), the present study identified STAT3 as a putative miR-17-5p target, including a plausible regulatory mechanism that may account for the modulation of GR. Indeed, the present study demonstrated that knockdown of miR-17-5p expression enhanced the expression of STAT3, thereby facilitating GR. However, the addition of $\mathrm{CuB}$ could upregulate miR-17-5p expression in GR PC9/GR cells, thereby ameliorating GR. Liu et al (5) reported that $\mathrm{CuB}$ could induce lysosomal stress-dependent death of GR NSCLC cells, which was consistent with the results of the present study.

It has been shown that $\mathrm{CuB}$ exhibits potent antineoplastic effects across a wide variety of cancer types, including colon (9), bladder (10), pancreatic (39), breast (40) and NSCLC $(41,42)$. It has been reported that miRNA can reverse the apoptotic effect of $\mathrm{CuB}$ on cancer cells (39). The present study demonstrated that inhibiting miR-17-5p negated the beneficial effects of $\mathrm{CuB}$ on PC9/GR cell responses to gefitinib, thereby upregulating proliferation, downregulating apoptosis and protein levels of apoptosis-related factors cleaved caspase-3 and cleaved caspase-9. This suggested that CuB-mediated miR-17-5p expression modulation impacted the proliferation and apoptosis of PC9/GR cells. As the underlying mechanisms remained unclear, it was identified via a TargetScan database analysis that the putative miR-17-5p target gene STAT3 may play a key role in such mechanisms.

STAT3 is a known oncogene. Specifically, its transcription promotes the occurrence and progression of NSCLC (43-45). Although the present study employed a dual-luciferase reporter assay to demonstrate that STAT3 is directly targeted by miR-17-5p, the impact of the miR-17-5p/STAT3 axis on PC9/GR cell function requires further verification. Findings of the present study also indicated that $\mathrm{CuB}$ dose-dependently enhanced STAT3 protein expression and phosphorylation, and during combined $\mathrm{CuB}$ and gefitinib (at $\mathrm{IC}_{25}$ ) treatment miR-17-5p negatively regulated STAT3 protein expression. Furthermore, overexpression of STAT3 increased proliferation and decreased apoptosis and protein levels of cleaved caspase- 3 and cleaved caspase- 9 of PC9/GR cells. These results indicated that miR-17-5p reversed the effect of $\mathrm{CuB}$ and gefitinib combination therapy in PC9/GR cells by activating the STAT3 protein. Notably, neither GR nor CUB treatment, nor miR-17-5p mimics or inhibitor transfection, affected the gene expression of STAT3 in PC9 or PC9/GR cells, suggesting that these factors could influence the post-transcriptional regulation of STAT3, but not the transcriptional level of STAT3. 

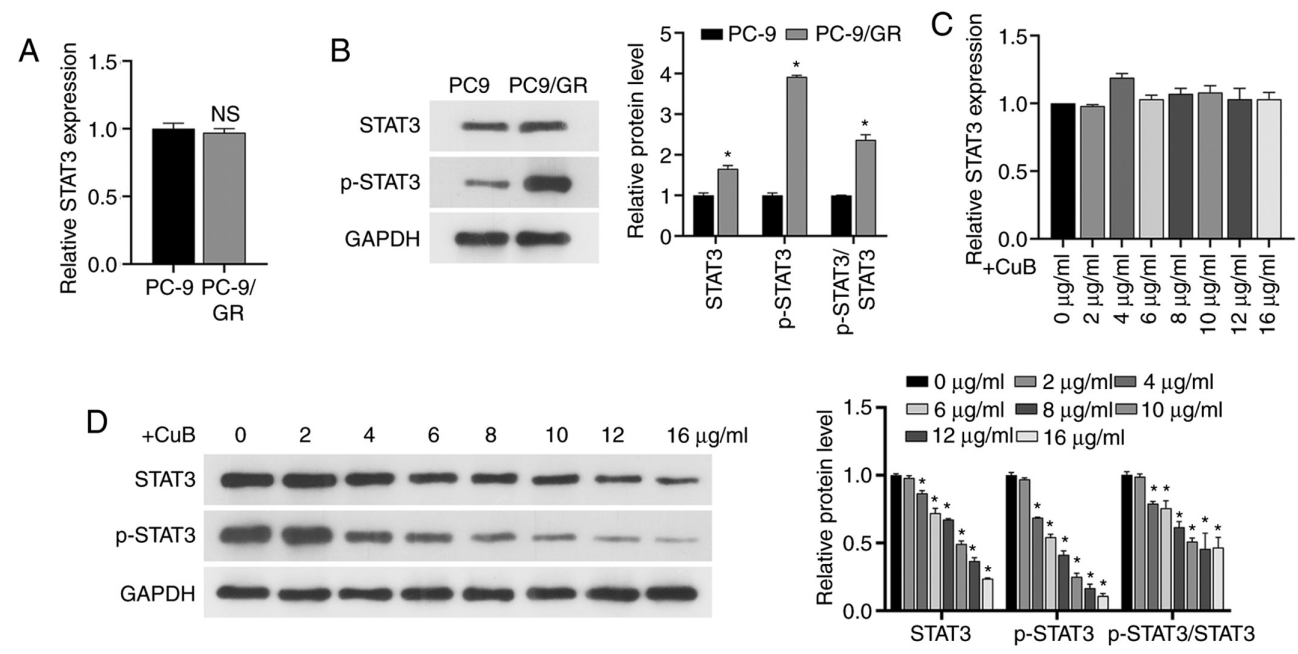

Figure 4. Effect of GR and CuB on STAT3 transcript and STAT3 protein levels in PC9/GR cells. (A) Transcript levels in PC9 and PC9/GR cells. (B) Protein expression levels of STAT3 and p-STAT3 in PC9 and PC9/GR cells. "P<0.05 vs. PC-9. (C) Impact of differing CuB concentrations on transcript levels in PC9/GR cells. (D) Impact of differing CuB concentrations on protein levels of STAT3 and p-STAT3 in PC9/GR cells. All values are expressed as mean \pm standard deviation. "P<0.05 vs. $0 \mu \mathrm{g} / \mathrm{ml}$. NS, not significant; CuB, cucurbitacin B; GR, gefitinib-resistant; p-, phosphorylated.
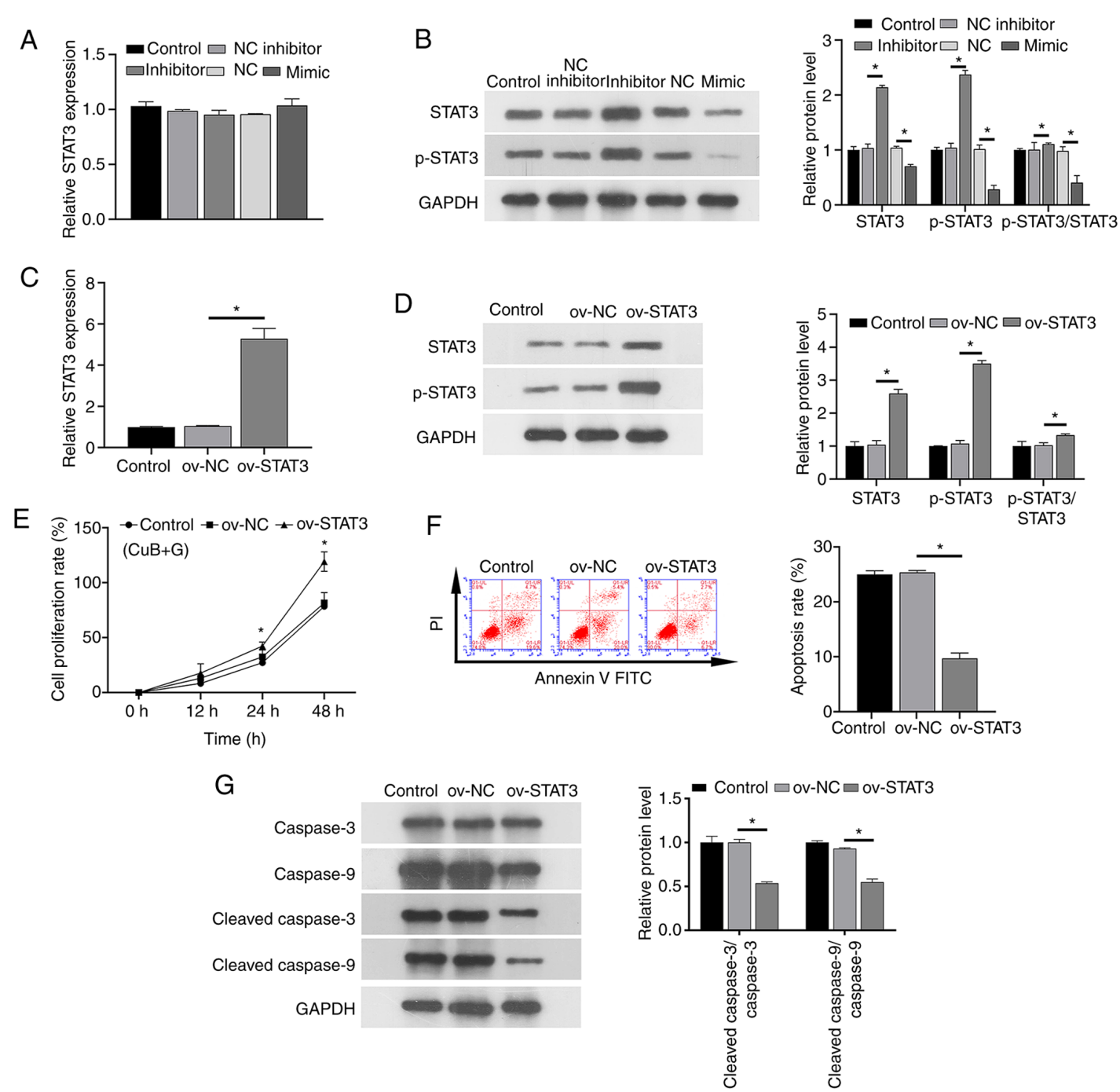

Figure 5. Effect of $\mathrm{CuB}$ in the presence of gefitinib at $\mathrm{IC}_{25}$ on PC9/GR cells transfected with miR-17-5p mimic or inhibitor, or overexpressing STAT3. (A) Impact of miR-17-5p mimic/inhibitor on STAT3 transcript levels. (B) Impact of miR-17-5p mimic/inhibitor on STAT3 protein levels. (C) Impact of STAT3 overexpression on STAT3 transcript levels. (D) Impact of STAT3 overexpression on STAT3 protein expression. (E) Impact of STAT3 overexpression on PC9/GR cell proliferation. (F) Impact of STAT3 overexpression on PC9/GR cell apoptosis. (G) Impact of STAT3 overexpression on caspase-3, cleaved caspase-3, caspase- 9 and cleaved caspase- 9 protein levels. All values are expressed as mean \pm standard deviation. "P<0.05. NS, not significant; CuB, cucurbitacin B; GR, gefitinib-resistant; NC, negative control; miR, microRNA; p-, phosphorylated; ov-, overexpression vector. 
This study has some shortcomings. First, there is no further discussion on related pathways. Second, this study did not analyze the combination of $\mathrm{CuB}$ and gefitinib in vivo to see a true reflection of the effects of this RNA-drug interaction in a complex biological system. These are also the goals of our future research.

In conclusion, $\mathrm{CuB}$ ameliorated NSCLC resistance to gefitinib by modulating the miR-17-5p/STAT3 axis in a manner that decreased STAT3 protein levels and phosphorylation, inhibited proliferation and promoted apoptosis. These initial preclinical findings supported this axis as a potential novel target for prevention or amelioration of GR. Further research is required to confirm that the beneficial effects of $\mathrm{CuB}$ persist in vivo, and such research should consider the associated biological pathways in more detail.

\section{Acknowledgements}

Not applicable.

\section{Funding}

This study was supported by the Natural Science Foundation of Guangdong Province, China (grant no. 2018A030310169).

\section{Availability of data and materials}

The datasets used and/or analyzed during the current study are available from the corresponding author on reasonable request.

\section{Authors' contributions}

YL designed the study and developed the methodology. BY, LZ and HT performed the experiments and collected the data. BY and WW analyzed and interpreted the data. BY drafted the original manuscript. YL and BY confirm the authenticity of all the raw data. All authors have read and approved the final manuscript.

\section{Ethics approval and consent to participate}

Not applicable.

\section{Patient consent for publication}

Not applicable.

\section{Competing interests}

The authors declare that they have no competing interests.

\section{References}

1. Wu YL, Cheng Y, Zhou J, Lu S, Zhang Y, Zhao J, Kim DW, Soo RA, Kim SW, Pan H, et al; INSIGHT Investigators: Tepotinib plus gefitinib in patients with EGFR-mutant non-small-cell lung cancer with MET overexpression or MET amplification and acquired resistance to previous EGFR inhibitor (INSIGHT study): An open-label, phase 1b/2, multicentre, randomised trial. Lancet Respir Med 8: 1132-1143, 2020.

2. Park K, Tan EH, O'Byrne K, Zhang L, Boyer M, Mok T, Hirsh V, Yang JC, Lee KH, Lu S, et al: Afatinib versus gefitinib as first-line treatment of patients with EGFR mutation-positive non-small-cell lung cancer (LUX-Lung 7): A phase 2B, open-label, randomised controlled trial. Lancet Oncol 17: 577-589, 2016.
3. Mitsudomi T, Morita S, Yatabe Y, Negoro S, Okamoto I, Tsurutani J, Seto T, Satouchi M, Tada H, Hirashima T, et al; West Japan Oncology Group: Gefitinib versus cisplatin plus docetaxel in patients with non-small-cell lung cancer harbouring mutations of the epidermal growth factor receptor (WJTOG3405): An open label, randomised phase 3 trial. Lancet Oncol 11: 121-128, 2010.

4. Lopez Sambrooks C, Baro M, Quijano A, Narayan A, Cui W, Greninger P, Egan R, Patel A, Benes CH, Saltzman WM, et al: Oligosaccharyltransferase inhibition overcomes therapeutic resistance to EGFR tyrosine kinase inhibitors. Cancer Res 78: 5094-5106, 2018.

5. Liu P, Xiang Y, Liu X, Zhang T, Yang R, Chen S, Xu L, Yu Q, Zhao H, Zhang L, et al: Cucurbitacin B induces the lysosomal degradation of EGFR and suppresses the CIP2A/PP2A/Akt signaling axis in gefitinib-resistant non-small cell lung cancer. Molecules 24: 24, 2019.

6. Noronha V,Patil VM, Joshi A, Menon N, Chougule A, Mahajan A, Janu A, Purandare N, Kumar R, More S, et al: Gefitinib Versus Gefitinib Plus Pemetrexed and Carboplatin Chemotherapy in EGFR-Mutated Lung Cancer. J Clin Oncol 38: 124-136, 2020.

7. Xia X, Liu Y, Liao Y, Guo Z, Huang C, Zhang F, Jiang L, Wang X, Liu J and Huang H: Synergistic effects of gefitinib and thalidomide treatment on EGFR-TKI-sensitive and -resistant NSCLC. Eur J Pharmacol 856: 172409, 2019.

8. Li L, Jiang L, Wang Y, Zhao Y, Zhang XJ, Wu G, Zhou X, Sun J, Bai J, Ren B, et al: Combination of metformin and gefitinib as first-line therapy for nondiabetic advanced NSCLC patients with EGFR mutations: A randomized, double-blind phase II trial. Clin Cancer Res 25: 6967-6975, 2019.

9. Dandawate P, Subramaniam D, Panovich P, Standing D, Krishnamachary B, Kaushik G, Thomas SM, Dhar A, Weir SJ, Jensen RA, et al: Cucurbitacin B and I inhibits colon cancer growth by targeting the Notch signaling pathway. Sci Rep 10: 1290,2020

10. Kurman Y, Kiliccioglu I, Dikmen AU, Esendagli G, Bilen CY, Sozen S and Konac E: Cucurbitacin B and cisplatin induce the cell death pathways in MB49 mouse bladder cancer model. Exp Biol Med (Maywood) 245: 805-814, 2020.

11. Li Y, Wang R, Ma E, Deng Y, Wang X, Xiao J and Jing Y: The induction of $\mathrm{G} 2 / \mathrm{M}$ cell-cycle arrest and apoptosis by cucurbitacin $\mathrm{E}$ is associated with increased phosphorylation of eIF2alpha in leukemia cells. Anticancer Drugs 21: 389-400, 2010.

12. Shukla S, Sinha S, Khan S, Kumar S, Singh K, Mitra K, Maurya R and Meeran SM: Cucurbitacin B inhibits the stemness and metastatic abilities of NSCLC via downregulation of canonical Wnt/ $\beta$-catenin signaling axis. Sci Rep 6: 21860, 2016.

13. Shukla S, Khan S, Kumar S, Sinha S, Farhan M, Bora HK, Maurya R, Meeran SM and Cucurbitacin B: Cucurbitacin B Alters the expression of tumor-related genes by epigenetic modifications in NSCLC and inhibits NNK-induced lung tumorigenesis. Cancer Prev Res (Phila) 8: 552-562, 2015.

14. Zhang Y, Li M and Hu C: Exosomal transfer of miR-214 mediates gefitinib resistance in non-small cell lung cancer. Biochem Biophys Res Commun 507: 457-464, 2018.

15. Zhang W, Lin J, Wang P and Sun J: miR-17-5p down-regulation contributes to erlotinib resistance in non-small cell lung cancer cells. J Drug Target 25: 125-131, 2017.

16. Chen Q, Si Q, Xiao S, Xie Q, Lin J, Wang C, Chen L, Chen Q and Wang L: Prognostic significance of serum miR-17-5p in lung cancer. Med Oncol 30: 353, 2013.

17. Chatterjee A, Chattopadhyay D and Chakrabarti G: miR-17-5p downregulation contributes to paclitaxel resistance of lung cancer cells through altering beclin1 expression. PLoS One 9: e95716, 2014.

18. Yu H, Lee H, Herrmann A, Buettner R and Jove R: Revisiting STAT3 signalling in cancer: New and unexpected biological functions. Nat Rev Cancer 14: 736-746, 2014.

19. Yang Y, Wang W, Chang H, Han Z, Yu X and Zhang T: Reciprocal regulation of miR-206 and IL-6/STAT3 pathway mediates IL6-induced gefitinib resistance in EGFR-mutant lung cancer cells. J Cell Mol Med 23: 7331-7341, 2019.

20. Bartel DP: MicroRNAs: Target recognition and regulatory functions. Cell 136: 215-233, 2009.

21. Shen M, Xu Z, Xu W, Jiang K, Zhang F, Ding Q, Xu Z and Chen Y: Inhibition of ATM reverses EMT and decreases metastatic potential of cisplatin-resistant lung cancer cells through JAK/ STAT3/PD-L1 pathway. J Exp Clin Cancer Res 38: 149, 2019.

22. Tong M, Wang J, Jiang N, Pan H and Li D: Correlation between p-STAT3 overexpression and prognosis in lung cancer: A systematic review and meta-analysis. PLoS One 12: e0182282, 2017. 
23. Gong WJ, Ma LY, Hu L, Lv YN, Huang H, Xu JQ, Huang DD, Liu RJ, Han Y, Zhang Y, et al: STAT3 rs4796793 contributes to lung cancer risk and clinical outcomes of platinum-based chemotherapy. Int J Clin Oncol 24: 476-484, 2019.

24. Zhou J, Kwak KJ, Wu Z, Yang D, Li J, Chang M, Song Y, Zeng H, Lee $\mathrm{LJ}, \mathrm{Hu} \mathrm{J}$, et al: PLAUR confers resistance to gefitinib through EGFR/P-AKT/survivin signaling pathway. Cell Physiol Biochem 47: 1909-1924, 2018.

25. Xu X, Li D, Liu J, Ma Z, Huang H, Min L, Dai L and Dong S: Downregulation of PTPRK promotes cell proliferation and metastasis of NSCLC by enhancing STAT3 activation. Anal Cell Pathol (Amst) 2019: 4265040, 2019.

26. Rokavec M, Öner MG, Li H, Jackstadt R, Jiang L, Lodygin D, Kaller M, Horst D, Ziegler PK, Schwitalla S, et al: IL-6R/STAT3/ miR-34a feedback loop promotes EMT-mediated colorectal cancer invasion and metastasis. J Clin Invest 124: 1853-1867, 2014.

27. Li H, Chen L, Li JJ, Zhou Q, Huang A, Liu WW, Wang K, Gao L, Qi ST and Lu YT: miR-519a enhances chemosensitivity and promotes autophagy in glioblastoma by targeting STAT3/Bcl2 signaling pathway. J Hematol Oncol 11: 70, 2018.

28. Du W, Pan Z, Chen X, Wang L, Zhang Y, Li S, Liang H, Xu C, Zhang Y, Wu Y, et al: By targeting Stat3 microRNA-17-5p promotes cardiomyocyte apoptosis in response to ischemia followed by reperfusion. Cell Physiol Biochem 34: 955-965, 2014

29. Liao XH, Xiang Y, Yu CX, Li JP, Li H, Nie Q, Hu P, Zhou J and Zhang TC: STAT3 is required for miR-17-5p-mediated sensitization to chemotherapy-induced apoptosis in breast cancer cells. Oncotarget 8: 15763-15774, 2017.

30. Ogino A, Kitao H, Hirano S, Uchida A, Ishiai M, Kozuki T, Takigawa N, Takata M, Kiura K and Tanimoto M: Emergence of epidermal growth factor receptor T790M mutation during chronic exposure to gefitinib in a non small cell lung cancer cell line. Cancer Res 67: 7807-7814, 2007

31. Livak KJ and Schmittgen TD: Analysis of relative gene expression data using real-time quantitative PCR and the 2(-Delta Delta C(T)) method. Methods 25: 402-408, 2001.

32. Peng W, He D, Shan B, Wang J, Shi W, Zhao W, Peng Z, Luo Q, Duan M, Li B, et al: LINC81507 act as a competing endogenous RNA of miR-199b-5p to facilitate NSCLC proliferation and metastasis via regulating the CAV1/STAT3 pathway. Cell Death Dis 10: 533,2019 .

33. Liu H, Wu Z, Zhou H, Cai W, Li X, Hu J, Gao L, Feng T, Wang L, Peng X, et al: The SOX4/miR-17-92/RB1 axis promotes prostate cancer progression. Neoplasia 21: 765-776, 2019.

34. Liu F, Zhang F, Li X, Liu Q, Liu W, Song P, Qiu Z, Dong Y and Xiang H: Prognostic role of miR-17-92 family in human cancers: Evaluation of multiple prognostic outcomes. Oncotarget 8 : 69125-69138, 2017.
35. Zhang X, Li Y, Qi P and Ma Z: Biology of miR-17-92 cluster and its progress in lung cancer. Int J Med Sci 15: 1443-1448, 2018.

36. Yang C, Jia X, Zhou J, Sun Q and Ma Z: The MiR-17-92 gene cluster is a blood-based marker for cancer detection in non-small-cell lung cancer. Am J Med Sci 360: 248-260, 2020.

37. Gong J, He L, Ma J, Zhang J, Wang L and Wang J: The relationship between miR-17-5p, miR-92a, and let-7b expression with non-small cell lung cancer targeted drug resistance. J BUON 22: 454-461, 2017.

38. Friedman RC, Farh KK, Burge CB and Bartel DP: Most mammalian mRNAs are conserved targets of microRNAs. Genome Res 19: 92-105, 2009.

39. Zhou J, Liu M, Chen Y, Xu S, Guo Y and Zhao L: Cucurbitacin B suppresses proliferation of pancreatic cancer cells by ceRNA: Effect of miR-146b-5p and lncRNA-AFAP1-AS1. J Cell Physiol 234: 4655-4667, 2019.

40. Dittharot K, Dakeng S, Suebsakwong P, Suksamrarn A, Patmasiriwat P, Promkan M and Cucurbitacin B: Cucurbitacin B Induces Hypermethylation of Oncogenes in Breast Cancer Cells. Planta Med 85: 370-378, 2019.

41. Marostica LL, de Barros ALB, Oliveira J, Salgado BS, Cassali GD, Leite EA, Cardoso VN, Lang KL, Caro MS, Durán FJ, et al: Antitumor effectiveness of a combined therapy with a new cucurbitacin $B$ derivative and paclitaxel on a human lung cancer xenograft model. Toxicol Appl Pharmacol 329: 272-281, 2017.

42. Marostica LL, Silva IT, Kratz JM, Persich L, Geller FC, Lang KL, Caro MS, Durán FJ, Schenkel EP and Simões CM: Synergistic antiproliferative effects of a new cucurbitacin B derivative and chemotherapy drugs on lung cancer cell line A549. Chem Res Toxicol 28: 1949-1960, 2015.

43. Zhang X, Sai B, Wang F, Wang L, Wang Y, Zheng L, Li G, Tang J and Xiang J: Hypoxic BMSC-derived exosomal miRNAs promote metastasis of lung cancer cells via STAT3-induced EMT. Mol Cancer 18: 40, 2019.

44. Hu W, Ru Z, Zhou Y, Xiao W, Sun R, Zhang S, Gao Y, Li X, Zhang $X$ and Yang H: Lung cancer-derived extracellular vesicles induced myotube atrophy and adipocyte lipolysis via the extracellular IL-6-mediated STAT3 pathway. Biochim Biophys Acta Mol Cell Biol Lipids 1864: 1091-1102, 2019.

45. Shi J, Li J, Yang S, Hu X, Chen J, Feng J, Shi T, He Y, Mei Z, He W, et al: LncRNA SNHG3 is activated by E2F1 and promotes proliferation and migration of non-small-cell lung cancer cells through activating TGF- $\beta$ pathway and IL-6/JAK2/STAT3 pathway. J Cell Physiol 235: 2891-2900, 2020. International (CC BY-NC-ND 4.0) License. 